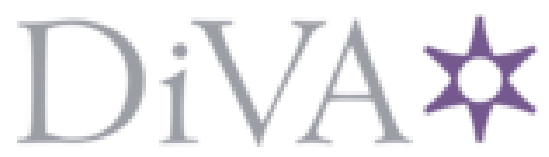

http://www.diva-portal.org

This is the published version of a paper presented at The 13th International Conference on Probabilistic Methods Applied to Power Systems; Durham, UK, July 7-10, 2014.

Citation for the original published paper:

Huang, Y., Alvehag, K., Söder, L. (2014)

Distribution network expansion planning considering distributed generation using probabilistic constraints.

In:

N.B. When citing this work, cite the original published paper.

Permanent link to this version:

http://urn.kb.se/resolve?urn=urn:nbn:se:kth:diva-148044 


\section{Distribution network expansion planning considering distributed generation using probabilistic voltage constraints}

Yalin Huang, Student member, IEEE, Karin Alvehag, Member, IEEE, and Lennart Söder, Member, IEEE

\begin{abstract}
A novel optimization model for network expansion planning, including distributed generation is proposed. The model considers the stochastic natures of distributed generation and load in the power systems. More importantly, this model addresses the probabilistic voltage constraints in the network expansion planning stage. The proposed model is employed to obtain the decisions for new wind power plant connections and reinforcements in the existing distribution network.
\end{abstract}

Index Terms-network expansion planning, distributed generation, reinforcement, connection, probabilistic voltage constraints.

\section{NOMENCLATURE}

1) Sets

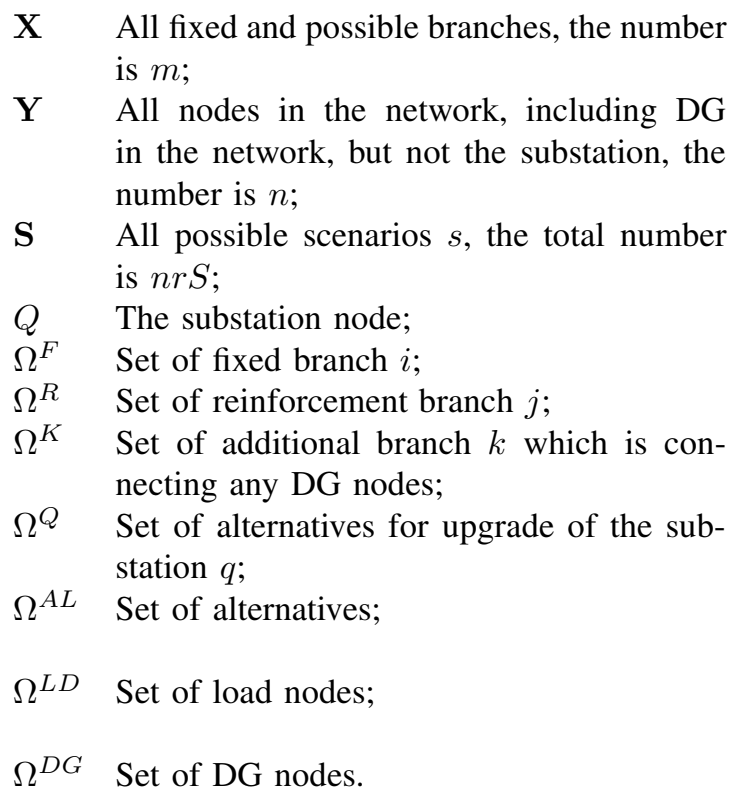

2) Parameters
$\mathbf{A}, \mathbf{A}^{\top}$
$n \times m$ node-branch incidence matrix and its transposition;
$P_{s}^{Y}, Q_{s}^{Y}$
Active and reactive power of nodal load in the scenario $s$;
$I_{s h, r e, s}^{Y} \quad$ Real part of the shunt current absorbed by nodal load in the scenario $s$;
$I_{s h, i m, s}^{Y} \quad$ Imaginary part of the shunt current ab- sorbed by nodal load in the scenario $s$;

$$
\begin{aligned}
& R_{j}^{A L}, X_{j}^{A L} \\
& R_{k}^{A L}, X_{k}^{A L} \\
& C_{j}^{A L}, O_{j}^{A L} \\
& C_{k}^{A L}, O_{k}^{A L}
\end{aligned}
$$$$
C_{q}^{A L}
$$$$
O_{i}
$$$$
V_{0}
$$$$
V_{\min }, V_{\max }
$$$$
I_{\max }^{X}, I_{\max }^{Q}
$$$$
P r_{e l}
$$$$
\operatorname{Prob}(s)
$$$$
\text { Prob }_{\text {volv }}
$$

3) Binary variables

Vector of resistance and reactance of the alternatives of the replacement branch $j$;

Vector of resistance and reactance of the alternatives of the additional branch $k$;

Vector of investment and operational costs of the alternatives of the replacement branch $j$;

Vector of investment and operational costs of alternatives of the additional branch $k$;

Fixed costs for substation installation of capacity $q$;

Vector of operational costs of fixed branch $i$;

Voltage at the substation;

Column vectors of the minimum and maximum voltage limits;

The maximum current limits on each branch and the substation;

The price of purchasing electricity from upstream grid;

The probability of each scenario $s$; The maximum probability of allowed voltage violation.

$\mathbf{D}_{j}^{A L}$

$\mathbf{D}_{k}^{A L}$

$\mathbf{D}_{q}^{A L}$

$N_{b r e a k_{l}, s}^{Y}$

$N_{b_{r e a k_{u}}, s}^{Y}$

4) Variables
Binary vector of decision investment on alternatives of the replacement branch $j$;

Binary vector of decision investment on alternatives of the additional branch $k$;

Binary vector of decision investment on alternatives of the upgrading substation $q$;

A binary variable indicates the voltage breaks the lower bound in scenario $s$;

A binary variable indicates the voltage breaks the upper bound in scenario $s$. 


$$
\begin{aligned}
& I_{x, r e, s}^{A L}, I_{x, i m, s}^{A L} \quad \text { Real part and imaginary } \\
& \text { part of current flows in } \\
& \text { any branch in scenario } \\
& s, \quad\left[I_{i, r e, s}^{A L}, I_{j, r e, s}^{A L}, I_{k, r e, s}^{A L}\right] \text {; } \\
& {\left[I_{i, i m, s}^{A L}, I_{j, i m, s}^{A L}, I_{k, i m, s}^{A L}\right] \text {; }} \\
& I_{0, r e, s}^{Q}, I_{0, i m, s}^{Q} \\
& V_{r e, s}^{Y}, V_{i m, s}^{Y} \\
& C^{c a p} \\
& C_{s}^{\text {oper }}
\end{aligned}
$$

\section{INTRODUCTION}

$\mathbf{M}$ ORE distributed wind power is expected to be connected to the distribution network and is considered to have the potential of improving system integrity, reliability and efficiency [1]. A lot of studies on distribution network planning considering generation uncertainties from wind power in a liberalized power industry have been done. However most of them are deterministic methods. Those methods require that the constraints should be satisfied with probability $100 \%$ in all scenarios (or only the pre-defined worst scenario), which is called stiff constraints in this paper. Wind power brings more intermittent production to the system, and to cope with this, some degree of violations of constraints could be tolerated in the planning stage. Therefore, it is reasonable to demand constraint satisfaction with some level of probability, which is called probabilistic constraints in this paper. This calls for the use of chance constrained optimization (CCOPT) in the distribution network planning model.

The major application areas of CCOPT and solution approaches are reviewed in [2]. It introduces the stateof-the-art in chance constrained nonlinear optimization problems. It concludes that evaluation of chance constraints becomes difficult when process models are nonlinear. A few publications have been found to apply CCOPT in electric power systems. Chance constrained programming (CCP) has been applied in transmission system expansion planning [3] [4]. The optimization problem using Monte Carlo simulation combined with genetic algorithm to solve the CCOPT. Only a few studies have considered the probabilistic power flow in analyzing hosting capacity and the impact from DG integration, e.g. [5], [6] and [7]. Furthermore, a few studies have considered the probabilistic voltage violation constraints in locating DG. Ref [8] formulates a multi-objective analysis of the optimal integration of wind turbines considering probabilistic voltage constraints. The analysis determines the optimal number of wind turbines to connect to the network and the optimal location of each of them.

However, to the authors' best knowledge, CCOPT has not been applied in distribution network planning considering distributed generation (DG) in a deregulated distribution system. Due to the deregulated rules, distribution system operators (DSOs) cannot invest in generation facilities. The model proposed in this paper formulates a chance constrained mix-integer optimization problem for distribution expansion planning under uncertainty. The new distribution network expansion planning model takes stochastic DG production and load profiles into consideration. It is expressed as a single objective stochastic optimization problem that minimizes the network cost while satisfying the constraints on the probabilistic nodal voltage violation constraint, the power capacity of distribution substations and feeders, the conditions on the radial networks etc.

\section{NETWORK PLANNING FORMULATION}

The optimization problem is formulated as a two-stage single objective mix-integer problem from a DSO's point of view. It aims to obtain the optimal long-term network expansion and reinforcement plan considering new wind power DG units for all scenarios of production and load. It is assumed that the regulation sends out incentives for DSOs to minimize the costs while guaranteeing technical requirements, and that DSOs can not own DG units but are obliged to connect them. This is, e.g., the case in Sweden, Italy and many countries where energy market is deregulated [9]. Thermal constraints, voltage rise or drop effects and short circuit currents rise effects are considered as the most relevant constraints to the longterm network planning with DG [10]. In this paper, thermal constrains and voltage rise limits are considered relevant for long-term network planning. In this model it is also assumed that the location and connection time of DG are known beforehand. Moreover, DG units are assumed to be connected at the same time, and several possible connection routes are predefined. Network expansion decisions, which reveal the optimal new DG connection routes, optimal conductors of the connection lines and reinforcement in the existing network, are represented by integer variables. These variables are stage-one variables since they do not vary with the scenarios. Voltages and currents in each scenario are stage-two variables because they vary with generation and load profiles.

\section{A. Expansion planning model}

The procedure is shown in Fig 1, where $k$ is the index for iteration, and $s$ is the index for scenarios. The optimization model starts with an assumed state of power system in which the voltages at all nodes in each scenario are $1 p . u$. as shown in Box A in Fig 1. Then the shunt currents of each load point in each scenario is calculated (Box B). The decision of lines should be made before the observation of all possible system states, therefore this is the stage-one variable in two-stage stochastic programming, the voltages of each node and currents on each line are the stage-two variables, which is scenario dependent as stated in Box C. The preliminary optimal network topology is obtained in Box $\mathrm{D}$, which is feasible in all scenarios and optimal in the total cost in average. The preliminary network topology, which defines the Y-matrix of the power system, is then the input 


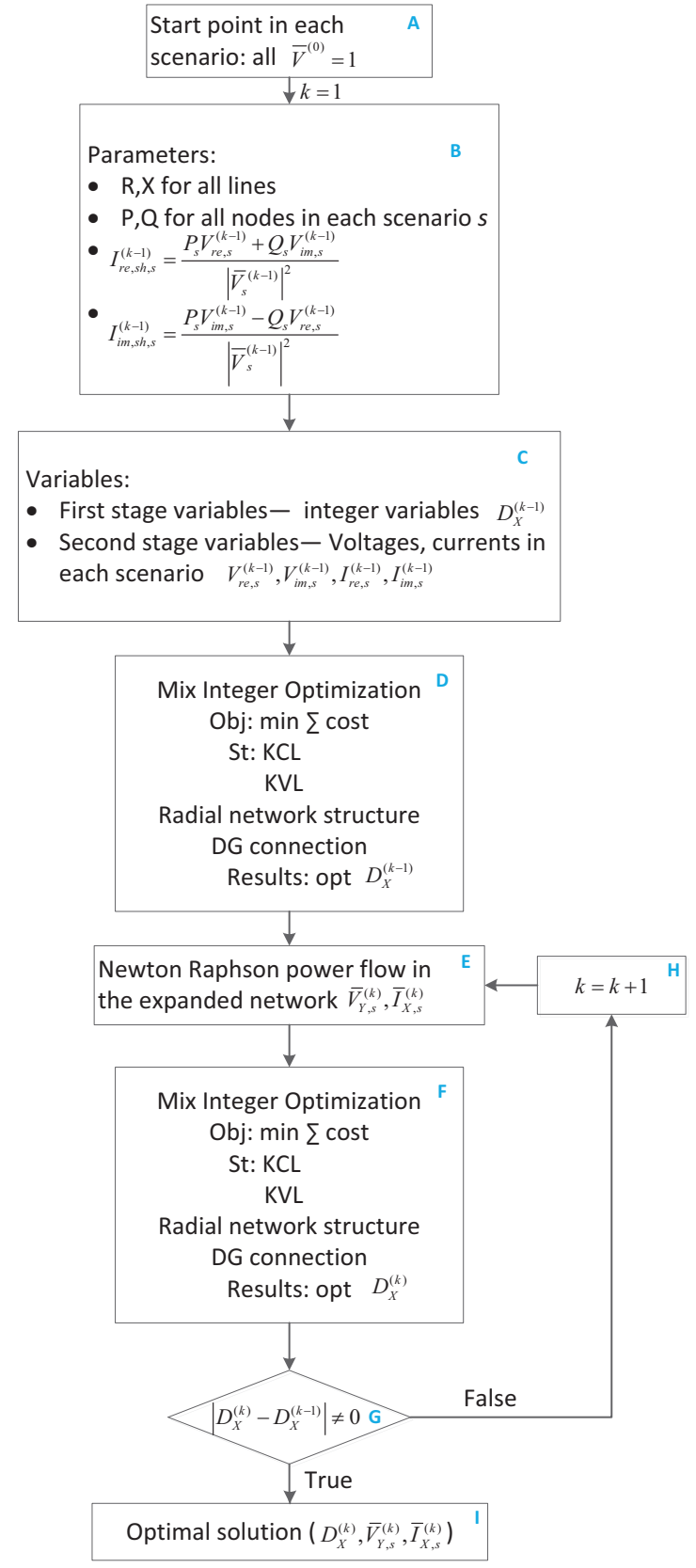

Fig. 1. Flowchart of the proposed method (KCL stands for Kirchhoff's current law; KVL stands for Kirchhoff's voltage law)

for the Newton Raphson power flow in each scenario in Box $\mathrm{E}$, therefore the "real" state (mainly the voltages and currents are relevant in this case) of the obtained network in each scenario is obtained. The results of the "real" state in each scenario are then used to re-do the optimization and obtain the new network (Box F, which is the same module as in Box D). If the decision results do not correspond to the previous results, go back to Box E and start the module of new power flow and optimization again. If the decision results match in two successive iteration, then the optimal expansion plan is obtained under the "real" states of the power system.
The planning model is developed in GAMS [11] and MATLAB, which allows simultaneously sophisticated optimization tools provided by GAMS with the user-friendly visualization tools provided by MATLAB. GAMS stands for general algebraic modeling system, which is very flexible at modifying the equations for optimization formulation. By storing the data and performing the iterative procedure in MATLAB, this model is problem-independent. By running Newton Raphson load flow in MATLAB, the non-linearity is avoided in the optimization problem. In Fig 1 only the mix integer optimization (Box D \& F) is performed in GAMS, while other parts are performed in MATLAB.

\section{B. Objective function}

This section presents the objective function of the optimization problem, which are in Box D (\& F) in Fig 1. The network is divided into three different parts: replacement branches, fixed network, additional branches. Replacement branches is the part of the network that would require reinforcement in the existing network. Fixed network is the remaining part of the existing network. Additional branches is the part of new connections in the network. This classification is useful to define different logical constraints in order to narrow down the searching area. The notations in this subsection relate to each iteration, so the iteration index is ignored.

The objective is to minimize the average total cost in the network for the long-term planning period considering production and load uncertainties. This paper considers the following cost functions:

- The installations cost of new lines and distribution substations

- The operational cost of the network

- The active power purchased from the upstream network

The operational cost and the power purchase are scenario dependent, however, the capital cost is only related to the decision variable. It is assumed that the operational cost of a line is proportional to the real part of the current on that line. This assumption is logical since the active power transmitted through the line is mainly related to the real part of the current due to the fact that voltage angles are small in the system. The current can flow in both directions in a system with DG units, thus the absolute value of the real part of the current is used. Furthermore, the cost of power exchange with the upstream grid is considered. Substation is the slack bus in the system; therefore, the cost is only calculated based on real part of the currents. If there is a reverse current flow (from nodes to the substation), it is considered as an income (negative cost). The net current flow inclusion in the objective also reflects losses in the downstream network since the amount of wind power and load are set in each scenario.

$$
C_{\text {total }}=C^{c a p}+\sum_{s} \operatorname{Prob}(s) * C_{s}^{o p e r}
$$




$$
\begin{aligned}
C^{c a p} & =\sum_{j} \mathbf{C}_{j}^{A L}\left(\mathbf{D}_{j}^{A L}\right)^{\top}+\sum_{k} \mathbf{C}_{k}^{A L}\left(\mathbf{D}_{k}^{A L}\right)^{\top} \\
& +\sum_{q} \mathbf{C}_{q}^{A L}\left(\mathbf{D}_{q}^{A L}\right)^{\top} \\
C_{s}^{\text {oper }} & =\sum_{i} O_{i}\left|I_{i, r e, s}\right|+\sum_{j} \mathbf{O}_{j}^{A L}\left|\mathbf{I}_{j, r e, s}^{A L}\right|^{\top} \\
& +\sum_{k} \mathbf{O}_{k}^{A L}\left|\mathbf{I}_{k, r e, s}^{A L}\right|^{\top}+\operatorname{Pr}_{e l} I_{0, r e, s}^{Q}
\end{aligned}
$$

In objective function (2b), the absolute value of the real part of the currents are used. Nonlinearity due to the absolute operation is linearized by introducing two non-negative variables: $I_{r e}^{p l u s}$ and $I_{r e}^{\text {minus }}$.

$$
\begin{aligned}
\left|I_{\text {re }}\right| & =I_{r e}^{\text {plus }}+I_{\text {re }}^{\text {minus }} \\
I_{\text {re }} & =I_{r e}^{\text {plus }}-I_{r e}^{\text {minus }}
\end{aligned}
$$

\section{Constraints}

The constraints to ensure a feasible and stable distribution network are:

i The radial configuration of distribution network

ii Kirchhoff's current law and voltage law (KCL \& KVL)

iii Deterministic thermal limits

iv Probabilistic voltage limits

$\mathrm{v}$ Connection of DG units to the existing network

Constraints (i) expresses the practical requirement from the DSO. In distribution systems it is more economical to build a radial network in most of the cases. Constraint (ii) is used to calculate the power flow for distribution network. Constraint (iii) ensures the line currents are within the limits all the time, while constraint (iv) ensures the probability of the average nodal voltage deviations are within a certain limit. Constraint (v) shows the optimal network expansion integrates the new DG units. In the following paragraphs, constraints (i) to (v) are explained further.

\section{i Radial configuration}

A radial feeder with $n+1$ nodes ( $n$ nodes for the network plus one node for the substation) always leads to a tree having at most $n$ branches [12]. $\operatorname{nr}(F)$ represents the number of fixed branches.

$$
\sum_{j, a l} \mathbf{D}_{j}^{A L}+\sum_{k, a l} \mathbf{D}_{k}^{A L}+n r(F) \leq N
$$

\section{ii Kirchhoff's circuit laws}

The physical balance of the system is guaranteed by KCL and KVL. KCL implies that the sum of currents flowing into a node should be equal to the sum of currents flowing out of it. KVL implies that the sum of the electrical voltage differences around any closed network is zero.

Equation (5a) applies to complex currents (both the real part and imaginary parts) in each scenario. It should be noted that the KVL only applies to the lines that exist in the network. The incidence matrix A converts the relationship between the nodes with all branches. Equation (5b) means that the real part of the voltage drop on each alternative of a line $x$ in each scenario, $\mathbf{R}_{x}^{A L} \mathbf{I}_{x, r e, s}^{A L}-\mathbf{X}_{x}^{A L} \mathbf{I}_{x, i m, s}^{A L}$, is equal to the real part of the voltage difference between two ends of the line, $\mathbf{A}^{\top} V_{r e, s}^{Y}$. Equation (5c) presents the imaginary part of voltage drops on a line. So the case in which no new line is built between two nodes should be excluded from (5b) and (5c). This condition can be implemented by multiplying the constraint by the corresponding decision variable. Nonlinearities would be thereby introduced into the model. This is, however, avoided by adopting an extension of the linear disjunctive model which is widely used in power system [12] [13] [14]. By introducing a big number $M,(5 \mathrm{~b})$ can be relaxed as (6), and (5c) follows the same manner.

$$
\begin{aligned}
\mathbf{A} \bar{I}_{s}^{X} & =\bar{I}_{s h, s}^{Y} \\
\mathbf{A}^{\top} \mathbf{V}_{r e, s}^{Y}-\mathbf{R}_{x}^{A L} \mathbf{I}_{x, r e, s}^{A L}+\mathbf{X}_{x}^{A L} \mathbf{I}_{x, i m, s}^{A L} & =\mathbf{0} \\
\mathbf{A}^{\top} \mathbf{V}_{i m, s}^{Y}-\mathbf{R}_{x}^{A L} \mathbf{I}_{x, i m, s}^{A L}-\mathbf{X}_{x}^{A L} \mathbf{I}_{x, r e, s}^{A L} & =\mathbf{0}
\end{aligned}
$$

where $\quad D_{j}^{A L}=1, \forall j \in \Omega^{R} \cup \Omega^{K}$

$$
\mathbf{A}^{\top} \mathbf{V}_{r e, s}^{Y}-\mathbf{R}_{x}^{A L} \mathbf{I}_{x, r e, s}^{A L}+\mathbf{X}_{x}^{A L} \mathbf{I}_{x, i m, s}^{A L} \leq M\left(1-\mathbf{D}_{x}^{A L}\right)
$$

It is the shunt currents $\bar{I}_{s h, s}^{Y}$ that are updated in each iteration as shown in Box E in Fig 1. The shunt current is calculated by (7b), which is formulated in a way suitable for the iteration process. The shunt admittance $\overline{\mathbf{Y}}_{s h}^{Y}$ at each node due to the contribution of the load is calculated by (7a). $\bar{S}_{s}=P_{s}^{Y}+j Q_{s}^{Y}$ is the complex power at each node (load and DG units). $\overline{\mathbf{Y}}$ is a diagonal matrix with the value $\overline{\mathbf{Y}}_{s h}^{Y}$.

$$
\begin{aligned}
\bar{Y}_{s h} & =\frac{\overline{S^{*}}}{V^{2}} \\
I_{s h, r e}+j I_{s h, i m} & =\bar{I}_{s h}=\overline{\mathbf{Y} \bar{V}}
\end{aligned}
$$

iii The thermal limits of substations and lines

The thermal limits of substations and lines are assumed stiff and no overloading is permitted. The current flowing through the substation is limited by the capacity of the substation. The capacity limit of the substation is represented by the current flowing through since the voltage is assumed to be $1 p . u$. at the substation. $n$ here represents the line that connected to the substation in (8a). The current limit on each line is assumed to be the real part of the current. It is also applied to the imaginary part of the current to force it to be zero when the line does not exit (i.e. the integer variable is zero).

$$
\begin{aligned}
\sum_{n} I_{n, s}^{Q} & \leq I_{\max }^{Q} \\
-I_{i, \max } \mathbf{D}_{i}^{A L} & \leq \mathbf{I}_{i, r e, s}^{A L} \leq I_{i, \max } \mathbf{D}_{i}^{A L} \\
-I_{i, \max } \mathbf{D}_{i}^{A L} & \leq \mathbf{I}_{i, i m, s}^{A L} \leq I_{i, \max } \mathbf{D}_{i}^{A L}
\end{aligned}
$$




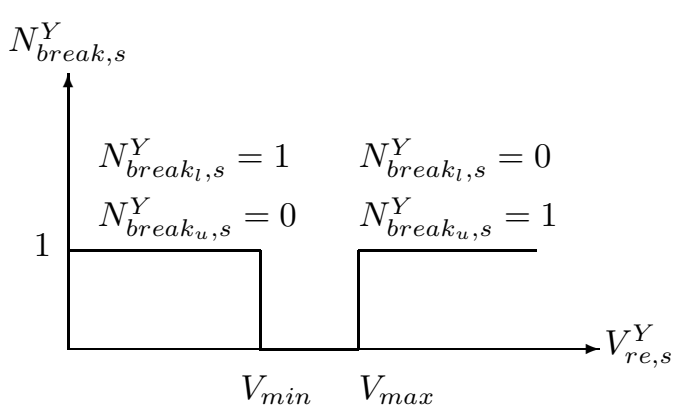

Fig. 2. Attributes measure the number of voltage violation

\section{iv The probabilistic voltage limits}

The voltage limits of each node is assumed probabilistic. The probability of voltage constrain violation is calculated as the ratio of the number of scenarios in which a node has a voltage violation over the number of total scenarios. Since the phase angles of the voltages are very small, the real part of it is used instead of the absolute value in the voltage limit constraints. Moreover, two binary variables are introduced to count the number of voltage violation of each bus. $N_{b_{r e a k_{l}, s}}^{Y}=1$ and $N_{\text {break }_{u}, s}^{Y}=0$ if the voltage of a node is less than the lower limit, while $N_{b r e a k_{u}, s}^{Y}=1$ and $N_{\text {break }_{l}, s}^{Y}=0$ if the voltage of a node is more than the upper limit in the scenario. Otherwise $N_{b r e a k_{u}, s}^{Y}=0$ and $N_{b r e a k_{l}, s}^{Y}=0$ if the voltage of the node is within the upper and lower bounds. This is shown in Fig 2.

It is expressed in linear equations:

$$
\begin{array}{r}
V_{r e, s}^{Y}-V_{\max } \geq\left(N_{\text {break }_{u}, s}^{Y}-1\right) * M \\
V_{r e, s}^{Y}-V_{\text {max }} \leq N_{\text {break }_{u}, s}^{Y} * M \\
V_{r e, s}^{Y}-V_{\min } \geq\left(N_{\text {break }_{l}, s}^{Y}-1\right) * M \\
V_{\text {re }, s}^{Y}-V_{\text {min }} \leq N_{\text {break }_{l}, s}^{Y} * M \\
N_{\text {break }_{u}, s}^{Y}+N_{\text {break }_{l}, s}^{Y}=N_{\text {break }, s^{\prime}}^{Y} \\
\frac{\sum_{s} N_{\text {break }, s^{Y}}^{Y} \leq \text { Prob }_{\text {volv }}}{n r S}
\end{array}
$$

The maximum probability of the voltage of a node being out of bounds depends on the voltage quality regulation. It is also possible to calculate the probability of any node in the system being out of bounds if it is necessary.

\section{$\mathrm{v}$ The logical constraints}

The model also takes logical constraints into account and predefines DG connection alternatives to reduce the search space. For the replacement branches, the sum of decisions on all alternatives should be not less than one. For the additional branches, the sum of decisions on all alternatives for connection one DG should be not less than one. If the optimal solution is two parallel lines, this means that the optimal solution is a line with the equivalent parameters (mainly resistance and reactance). If in reality the choices are limited, then these logical constraints can be changed respectively.

$$
\begin{aligned}
& \sum_{a l} \mathbf{D}_{j}^{A L} \geq 1 \\
& \sum_{a l} \mathbf{D}_{k^{\prime}}^{A L} \geq 1 \\
& \sum_{a l} \mathbf{D}_{q}^{A L}=1
\end{aligned}
$$

where $k^{\prime}$ represents all the routes of connection one DG units.

\section{CASE STUDY}

To validate the mathematical model given in Section II a fictitious distribution network with wind power plant connections is applied. Multi-scenarios of production and consumption in the network are studied, the network expansion decision is made from probabilistic voltage constraints. At the same time, the network expansion decision based on the stiff voltage constraints are showed.

The computation is performed in a PC Intel Core i7 2.70 $\mathrm{GHz}, 8 \mathrm{~GB}$ RAM. The iterative procedure is performed in MATLAB 7.11 while the mixed integer optimization procedure is performed in GAMS 23.9 using CPLEX solver.

\section{A. Background}

The network in this case study is a distribution system operating at $24900 \mathrm{~V}$ with 21 load nodes spread over the area. It is displayed in Fig 3. In this figure, the square node represents the feeding substation, the circles are the nodes and the wind turbine represents a wind farm. The branches between nodes represent the electrical connections between nodes. Continuous lines denote the existing network and dashed lines are candidate routes for new connections. The basic values for the whole network are 2.5 MVA and 24.9 $\mathrm{kV}$. Two wind farms are in the pipeline to be connected, six possible connection routes are predefined.

The production and consumption at each node are assumed to follow normal distributions. In the case of applying probabilistic voltage constraints, 20 scenarios from the distribution function are randomly chosen. Applying the European standard EN 50160 [15], voltages should stay within the range of $\pm 10 \%$ of the nominal voltage during $95 \%$ of the time of one week. In this case study, voltage limits are set as 0.9 and 1.1 of the nominal voltage, and in $95 \%$ of the scenarios voltages on all nodes are ensured to respect the limits. The operation and maintenance cost of all branches are assumed to be 1 and proportional to the absolute currents. The price for the purchasing electricity from upstream grid is assumed to be $100 /$ p.u.

\section{B. Results}

The results of decision for expanding the network with DG units by probabilistic voltage constraints and stiff voltage 


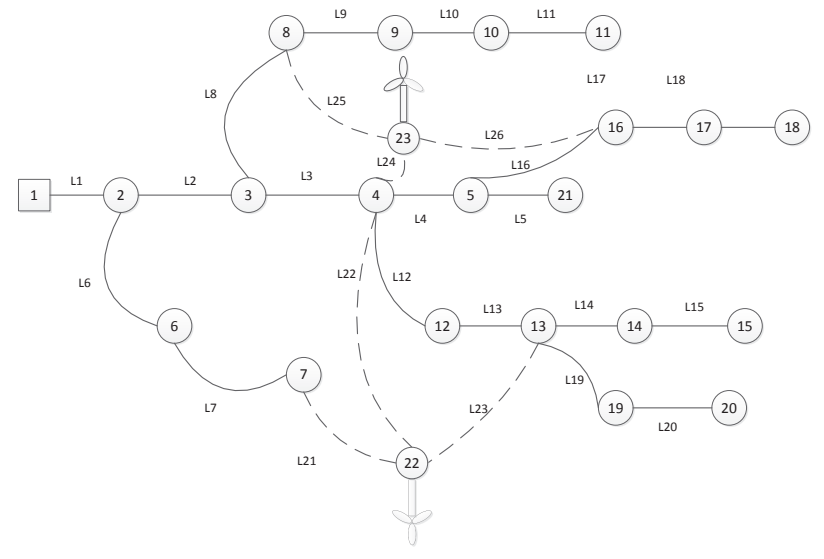

Fig. 3. Diagram of the 23-node network

constraints are shown in Table I.

The expansion plan using probabilistic voltage constraints shows that $L 21$ and $L 22$ are needed to be built to connect wind farm on node 22, while L25 and L26 are needed to be built to connect wind farm on node 23 . The cost is 111 . The execution time is 29.9 seconds and it converges after six iterations. The expansion plan using stiff voltage constraints shows that the same paths need to be build to connect the new wind power plants but different line conductors. The cost is 119. The execution time is 10.7 seconds and it converges after three iterations.

TABLE I

RESUlTS FOR DECISIONS IN CASE STUDY

\begin{tabular}{llllllllll}
\hline \hline Decision & $L 1$ & $L 2$ & $L 3$ & $L 4$ & $L 5$ & $L 6$ & $L 7$ & $L 8$ & $L 9$ \\
\hline AL(prob.) 1 & 1 & 1 & 1 & 1 & 1 & 1 & 1 & 1 \\
AL(stiff) & 1 & 1 & 1 & 1 & 1 & 1 & 1 & 1 & 1 \\
\hline \hline Decision & $L 10$ & $L 11$ & $L 12$ & $L 13$ & $L 14$ & $L 15$ & $L 16$ & $L 17$ & $L 18$ \\
\hline AL(prob.) & 1 & 1 & 1 & 1 & 1 & 1 & 1 & 1 & 1 \\
AL(stiff) & 1 & 1 & 1 & 1 & 1 & 1 & 1 & 1 & 1 \\
\hline \hline Decision & $L 19$ & $L 20$ & $L 21$ & $L 22$ & $L 23$ & $L 24$ & $L 25$ & $L 26$ & \\
\hline AL(prob.) & 1 & 1 & 2 & 3 & 1 & 0 & 3 & 2 & \\
AL(stiff) & 1 & 1 & 2 & 3 & 1 & 0 & 3 & 3 & \\
\hline
\end{tabular}

\section{CONCLUSION}

A new method for optimal expansion planning of radial distribution networks using probabilistic voltage constraints is proposed. The method is also able to take DG units which are not owned by the DSO into account. Cost of planning with probabilistic voltage constraints and the stiff voltage constraints are compared. The case study clearly shows that considering voltage constraints probabilistically in the planning stage decreases the cost of the system. Moreover, the proposed method is able to consider different alterations to distribution networks for DSOs in unbundled market where DG units are not sited by the DSOs. Furthermore, this method requires low computational time. The mathematical optimization formulation shows its flexibility on adapting technical constraints, which contributes to a reduction in the search space. The low computation time is also due to the cooperation between MATLAB and GAMS. MATLAB can efficiently handle the nonlinear power flow in the network expansion planning problem, which makes the optimization model in GAMS linear and consequently faster.

\section{REFERENCES}

[1] M. Nehrir, C. Wang, and V. Gerez, "Impact of wind power distributed generation on distribution systems," in Proc. IEEE 17th Int. Conf. Electricity Distribution (CIRED). Barcelona, Spain, May 2003.

[2] A. Geletu, M. Klöppel, H. Zhang, and P. Li, "Advances and applications of chance-constrained approaches to systems optimisation under uncertainty," International Journal of Systems Science, vol. 44, no. 7, pp. 1209-1232, 2013.

[3] N. Yang and F. Wen, "A chance constrained programming approach to transmission system expansion planning," Electric Power Systems Research, vol. 75, no. 23, pp. 171 - 177, 2005.

[4] H. Yu, C. Chung, K. Wong, and J. Zhang, "A chance constrained transmission network expansion planning method with consideration of load and wind farm uncertainties," Power Systems, IEEE Transactions on, vol. 24, no. 3, pp. 1568-1576, 2009.

[5] G. Papaefthymiou, P. H. Schavemaker, L. van der Sluis, W. L. Kling, D. Kurowicka, and R. M. Cooke, "Integration of stochastic generation in power systems," International Journal of Electrical Power \& Energy Systems, vol. 28, no. 9, pp. 655-667, Nov. 2006.

[6] L. F. Rocha and C. L. T. Borges, "Probabilistic generation and interruption costs and other economic aspects related to Distributed Generation integration," in IEEE PES General Meeting. IEEE, Jul. 2010, pp. 1-6.

[7] F. J. Ruiz-Rodriguez, J. C. Hernández, and F. Jurado, "Probabilistic load flow for photovoltaic distributed generation using the CornishFisher expansion," Electric Power Systems Research, vol. 89, pp. 129-138, Aug. 2012.

[8] A. Alarcon-Rodriguez and G. W. Ault, "Multi-objective planning of distributed energy resources with probabilistic constraints," in Power and Energy Society General Meeting, 2010 IEEE, 2010, pp. 1-7.

[9] Y. Huang, "Market concepts and regulatory bottlenecks for smart distribution grids in eu countries," Master's thesis, KTH, Electric Power Systems, 2011.

[10] X. Tao and H.-J. Haubrich, "A Two-Stage Heuristic Method for the Planning of Medium Voltage Distribution Networks with Large-Scale Distributed Generation," in International Conference on Probabilistic Methods Applied to Power Systems, IEEE. Stockholm, Sweden, Jun. 2006, pp. 1-6.

[11] A. Brooke, D. Kendrick, A. Meeraus, R. Raman, and R. Rosenthal, "Gams a user's guide (gams development corporation, washington dc)," 1998.

[12] S. Haffner, L. F. A. Pereira, L. A. Pereira, and L. S. Barreto, "Multistage Model for Distribution Expansion Planning With Distributed Generation ;Part I: Problem Formulation," IEEE Transactions on Power Delivery, vol. 23, no. 2, pp. 915-923, Apr. 2008.

[13] L. Bahiense, G. C. Oliveira, M. Pereira, and S. Granville, "A mixed integer disjunctive model for transmission network expansion," IEEE Transactions on Power Systems, vol. 16, no. 3, pp. 560-565, 2001.

[14] R. S. Agniar and P. Cuervo, "Capacitor placement in radial distribution network through a linear deterministic optimization model," 2005.

[15] H. Markiewicz and A. Klajn, "Voltage disturbances standard en 50160 - voltage characteristics in public distribution systems," no. ENYARTICLE-2008-194, p. 16p, jul. 2004. 